\title{
Cariotipos de los caracoles de tinte Plicopurpura pansa y Plicopurpura columellaris (Gastropoda: Muricidae)
}

\author{
Lenin Arias-Rodriguez ${ }^{1,3}$, Juan P. González-Hermoso², Horacio Fletes-Regalado², Luz Estela \\ Rodríguez-Ibarra $^{1} \&$ Gabriela Del Valle Pignataro ${ }^{1}$ \\ 1 Centro de Investigación en Alimentación y Desarrollo, A.C. Unidad, Mazatlán, Sábalo-Cerritos S/N Estero del Yugo, \\ A.P. 711, Mazatlán, Sinaloa, México. Tel. (669) 989-87-00. Fax (669) 989-87-01; leninariasrodriguez@hotmail.com \\ 2 Universidad Autónoma de Nayarit, Facultad de Ingeniería Pesquera, Bahia de Matanchan, Km 12, Carretera los \\ Cocos, A.P. 10, San Blas Nayarit, Mexico. Tel/Fax. (323) 31-21-20. \\ 3 División Académica de Ciencias Biológicas, UJAT. Carretera Villahermosa-Cárdenas Km $0.5 \mathrm{~S} / \mathrm{N}$. Entronque a \\ Bosques de Saloya. C.P. 86150. Tel. (993) 354-4308. Villahermosa, Tabasco, México.
}

$$
\text { Recibido 04-III-2006. Corregido 11-XII-2006. Aceptado 14-V-2007. }
$$

\begin{abstract}
Karyotypes of the purple snails Plicopurpura pansa and Plicopurpura columellaris (Gastropoda: Muricidae). The karyotypes of the purple snails Plicopurpura pansa (Gould, 1853) and P. columellaris (Lamarck, 1816) were established from 17 and 13 adults, respectively; and from eight capsules with embryos of P. pansa. In P. pansa were counted 59 mitotic fields in the adults and 127 in embryos; and 118 fields in $P$. columellaris. Chromosome numbers from 30 to 42 were observed in both species. Such a variation was notorious in each sample and there was no evidence of any relationship with tissue (gill, muscle and stomach). Both species has a typical modal number of $2 \mathrm{n}=36$ chromosomes. Five good quality chromosome spreads were selected from adults of each species to assemble the karyotype. Classic cytogenetics statistics like relative lengths, arm ratio, centromeric index and the difference between long and short arms are presented. There were three pairs of metacentric and fifteen pairs of telocentric chromosomes in both species. This classification was not strong enough, so the chromosome complement by species was divided in four groups ("a", "b", "c" and "d") on the basis of relative lengths $(\mathrm{p}+\mathrm{q})$. A comparison of $\mathrm{p}+\mathrm{q}$ in each chromosome pair was estimated within and between species by two ways analysis of variance and Tukey tests $(P<0.05)$. Significant differences were identified among chromosome groups in each species; the differences between species were given by the first three pairs of chromosomes (group "a" biarmed) and the last two pairs (group "d" uniarmed). Deviations in chromosome number and relative lengths probably are given by chromosome rearrangements, related with chromosome polymorphism and presence of the atypical microchromosome "B". The fundamental number in both species was characterized by 42 chromosome arms. No sex chromosomes were identified. Rev. Biol. Trop. 55 (3-4): 853-866. Epub 2007 December, 28.
\end{abstract}

Key words: Plicopurpura, pansa, columellaris, chromosomes, polymorphism.

En las costas de México se ha reportado la presencia de tres especies del género Plicopurpura o caracoles de tinte; dos de ellas, $P$. pansa y $P$. Columellaris, habitan en el pacífico y $P$. patula en la región golfo-Pacífico (Castillo-Rodriguez 1992a, b). El límite geográfico de ambas especies es Baja California en el norte de México (Keen 1971) y en el sur hasta las costas del Perú, Chile y las Islas Galápagos (Turok et al. 1988, Turok 1999).
Dichas especies, han tenido importancia en las tradiciones y ceremonias de varios grupos indígenas de la región pacifico de México y Sudamérica, desde la época precolombina hasta la actual (Turok et al. 1988, Castillo-Rodriguez 1992a). En lo concerniente a investigaciones realizadas en el género Plicopurpura, éstas se han enfocado a observaciones de tipo poblacional y algunas relacionadas con el crecimiento (Acevedo y Hernández 1987, Álvarez 1989, 
Castillo-Rodriguez 1992b, Michel-Morfin et al. 2000, 2002, Ramirez-Rodriguez y Naegel 2003). Aspectos de carácter anatómico y morfológico han sido evaluados con el propósito de resolver conflictos de carácter taxonómico en el género (Clench 1947, Abbott 1974, Kool 1987, 1988, Castillo-Rodriguez 1992a); o como en el caso de $P$. pansa para describir el ciclo reproductivo y desarrollo embrionario de la especie en cautiverio (Naegel 2004).

La composición química de los pigmentos fue evaluada por Wouters (1992), así también la posible explotación comercial (Rios-Jara et al. 1994, Michel-Morfin et al. 2002).

Los caracteres conquiliológicos y anatómicos de las especies nominales $P$. pansa, $P$. patula y $P$. columellaris de acuerdo con Castillo-Rodriguez (1992a) integran un grupo interespecífico morfológicamente. Dichas observaciones, se han identificado durante el desarrollo experimental del cultivo de $P$. pansa y $P$. columellaris. En $P$. pansa, las conchas toman coloración y textura diferente a lo reportado en la descripción taxonómica representativa de la especie (Wellington y Kuris 1983).

Conflictos de carácter taxonómico utilizando métodos clásicos de identificación han sido reportados con frecuencia en la literatura relacionada con la familia Thaididae y Muricidae (Cernohorsky 1969, Keen 1971, Kool 1987, 1988, Castillo-Rodriguez 1992a). A raíz de dichos estudios, se ha sugerido la asignación específica a la familia Thaididae y/o Muricidae sin llegar a un acuerdo válido. Recientemente, Castillo-Rodriguez (1992a) sugiere que $P$. pansa, $P$. columellaris y $P$. patula se sitúen dentro de la unidad taxonómica de la familia Muricidae, sugerencia que fue adoptada en este documento.

El conocimiento de las adaptaciones y las relaciones de carácter evolutivo entre los miembros del grupo son aun desconocidas (Turok et al. 1988). La información relacionada con aspectos citogenéticos en el género Plicopurpura no existen (Thiriot-Quiévreux 2003) y los estudios citogenéticos reportados en el orden Neogastropoda han sido escasos, con reportes en solo 10 familias del grupo (Thiriot-Quiévreux 2003).

En la familia Muricidae, los números cromosómicos en estado diploide varían de 26-36 cromosomas (Dixon et al. 1994, Pascoe y Dixon 1994, Page 1988). Por ejemplo, en Nucella lapillos, dichos autores señalan que las variaciones en el número de cromosomas es el resultado de un alto grado de polimorfismo Robertsoniano asociado a la presencia de cromosomas tipo "B". En N. canaliculata, N. lima y $N$. lamellosa se ha registrado como número análogo 70 cromosomas (Ahmed 1974). Mientras que en la condición haploide en Murex brandaris y M. trunculus, números de 35 cromosomas (Ahmed 1974, Vitturi et al. 1982). En el caso de los miembros de la familia Thaididae los reportes no existen (ThiriotQuiévreux 2003).

En plantas y animales han sido ampliamente documentados la caracterización de cromosomas pequeños o microcromosomas y la de cromosomas grandes o de tamaño estándar; ambos adicionales al complemento cromosómico diploide (2n) del cariotipo típico (White 1954, Jones y Rees 1982, Beukeboom 1994, Camacho et al. 2000, Palestis et al. 2003, 2004, Granado et al. 2004, Moreira-Filho et al. 2004). La observación de variaciones en el número o tamaño de los cromosomas que integran un cariotipo, se ha relacionado con la presencia de microcromosomas y/o cromosomas tipo "B" (Jones y Rees 1982, Beukeboom 1994, Camacho et al. 2000, Palestis et al. 2003, 2004, Granado et al. 2004, MoreiraFilho et al. 2004). Los cromosomas "B", son cromosomas indispensables y adicionales al complemento estándar (cromosomas "A"), con frecuencia son heterocromáticos (fragmentos de $\mathrm{ADN}$ repetitivo que es transcripcionalmente inactivo), y no contienen genes importantes (Jones y Rees 1982, Rebollo y Arana 2001, Fagundes et al. 2004, Granado et al. 2004). Aunque están integrados en algunos casos por secuencias de ADN ribosomal, no muestran segregación mendeliana por lo que no se aparean y recombinan con los cromosomas "A" 
durante la meiosis (Jones y Rees 1982, Rebollo y Arana 2001, Fagundes et al. 2004, Granado et al. 2004). No obstante, se ha observado que es posible la integración o fusión de un cromosoma " $B$ " en un cromosoma "A" estándar; por ejemplo en los cromosomas "A" $\mathrm{y}$ "B" del grillo Eyprepocnemis ploran se ha discutido ampliamente dicho proceso (Granado et al. 2004). Por otro lado, ambos tipos de cromosomas se han observado con un alto grado de polimorfismo con variación y frecuencia considerable en las poblaciones naturales. Dicha variación en el número de cromosomas " $\mathrm{B}$ " $\mathrm{y} / \mathrm{o}$ en el tamaño de los mismos se han identificado a nivel individual (variación intra-individual) o con variaciones entre individuos (variación inter-individual) de una especie (Jones y Rees 1982, Beukeboom 1994, Camacho et al. 2000, Palestis et al. 2003, 2004, Granado et al. 2004, Moreira-Filho et al. 2004).

El polimorfismo cariotípico, se ha documentado en una variedad importante de especies animales, involucrando diferentes clases de rearreglos cromosómicos (White 1954). Por ejemplo el polimorfismo Robertsoniano encontrado en N. lapillos (Page 1988, Dixon et al. 1994, Pascoe y Dixon 1994).

En los miembros del género Plicopurpura, se han realizado estudios en taxonomía, ecología, anatomía, reproducción y desarrollo embrionario. Sin embargo, en el género fueron desestimados estudios relacionados con el análisis del número de cromosomas y la estructura de los mismos. Aunque son una herramienta importante para el diagnóstico de relaciones de tipo filogenético y de biología básica. Además, son primordiales en estudios complementarios de biología básica como filogenia y la de caracterizar procesos evolutivos para resolver conflictos de carácter taxonómico en especies con hábitat simpátrico como es el caso de las especies señaladas en este estudio.

El propósito de este análisis fue el de proveer una herramienta complementaria para la comprensión de la biológica básica y la taxonomía de las especies $P$. pansa y $P$. columellaris en México. Por ello, se reporta por primera ocasión el número cromosómico y la estructura morfológica de los cromosomas que constituyen el cariotipo típico de los caracoles de tinte.

\section{MATERIALES Y MÉTODOS}

Área de recolecta y caracterización de los especímenes: fueron recolectados y procesados 30 especímenes adultos en "Playa Platanitos" municipio de Compostela, estado de Nayarit, México. Diecisiete especímenes de $P$. pansa (12 machos y cinco hembras) y 13 de $P$. columellaris (nueve machos y cuatro hembras). Los organismos fueron mantenidos en tanques de fibra de vidrio con agua de mar tratada con $\mathrm{UV}$, temperatura aproximada de $20.0 \pm 1.0{ }^{\circ} \mathrm{C}$, salinidad de $35 \mathrm{ppm}$ y aireación constante. La alimentación consistió de una dieta a partir de calamar fresco ( $2 \mathrm{~g} /$ individuo) cada tercer día. Los organismos fueron identificados y separados por especie y sexo, de acuerdo con el criterio clásico de Clench (1947), Keen (1971) y Abbott (1974). Así como los caracteres conquiliológicos específicos citados por Kool (1987, 1988) y los caracteres radulares y anatómicos descritos por Castillo-Rodríguez (1992a).

Procedimiento citogenético: los caracoles adultos fueron inyectados con $50 \mu \mathrm{g}$ de colchicina por gramo de peso del ejemplar, diluyendo el alcaloide al $1.0 \%$ en una solución con $0.1 \%$ de citrato de sodio. Para aplicar la inyección, se hizo una abertura pequeña en la parte baja de la concha, sobre la espira de la región sutural (canícula) utilizando una segueta de acero. El tratamiento con colchicina mas apropiado fue de $10 \mathrm{~h}$ y los organismos fueron mantenidos con aireación constante en un contenedor con agua de mar filtrada. Adicionalmente, fueron utilizadas ocho cápsulas obtenidas de la reproducción en cautiverio de $P$. pansa en la Facultad de Ingeniería Pesquera de la Universidad Autónoma de Nayarit. Las cápsulas contenían aproximadamente de 800 a 900 embriones y fueron tratadas por seis horas con una solución de colchicina al $0.05 \%$ diluida en agua de mar. Los organismos adultos fueron sacrificados por hipotermia (exposición a hielo 
por $10 \mathrm{~min}$ ) y se extrajeron branquias, músculo, estómago y gónadas. Posteriormente, se colocaron a temperatura ambiente $\left(25.0 \pm 1.0^{\circ} \mathrm{C}\right)$ en una solución de citrato de sodio al $2.0 \%$ para hidratar por $60 \mathrm{~min}$ las células de cada tejido.

Los tejidos fueron disgregados para aumentar la exposición al agente hidratante. Cada tejido fue colocado en viales de $2 \mathrm{ml}$ y centrifugado a 5000 r.p.m. durante cinco minutos. Los restos de citrato de sodio fueron sustituidos por fijador, mezclando metanol frío $\left(4{ }^{\circ} \mathrm{C}\right)$ y ácido acético en proporción 4:1 cada $10 \mathrm{~min}$. Los recambios del fijador se realizaron seis veces ó hasta que el fijador se tornara transparente y los tejidos tomaran coloración blanquecina. En el caso de las muestras provenientes de los embriones, solo se realizaron tres cambios del fijador. Posteriormente, fueron conservadas a $-40{ }^{\circ} \mathrm{C}$ por dos meses. Para elaborar las laminillas, portaobjetos limpios fueron inmersos en alcohol etílico al $70 \%$ y mantenidos en refrigeración $\left(-20^{\circ} \mathrm{C}\right)$ por $2 \mathrm{~h}$, consecutivamente se escurrieron para el goteo de las células fijadas desde una altura aproximada de $1.80 \mathrm{~m}$.

En los embriones, lotes de aproximadamente 100 individuos fueron disgregados en el fijador 4:1 con el propósito de elaborar las laminillas. En todos los casos las laminillas fueron secadas con un mechero de alcohol (Denton 1973, Thorgaard y Disney 1990). Las preparaciones cromosómicas se envejecieron en una estufa termo regulada a $45{ }^{\circ} \mathrm{C}$ por 1 $\mathrm{h}$, con el fin de lograr una tinción adecuada por 10 min con una solución de giemsa al $10 \%$ preparada con fosfato buffer $\mathrm{pH} 7.0$ (Kligerman y Bloom 1977).

Elaboración del cariotipo y análisis estadístico: la búsqueda de las dispersiones cromosómicas se realizó con los objetivos 10X, 40X y 100X de un microscopio Olimpus CX 31 y las mejores dispersiones (menos encimadas y contraídas) fueron foto digitalizadas con un microscopio DMLB y cámara MPS 30 Leica. El número cromosómico modal diploide fue determinado por conteo directo bajo el microscopio y de fotografías de campos con cromosomas dispersos y no encimados.
Para construir el cariotipo, se ampliaron y recortaron cinco de las mejores fotografías de los adultos de cada especie, los cromosomas fueron colocados en orden de longitud y de acuerdo a la posición del centrómero. Se tomaron las medidas de longitud total de los brazos p (brazo corto) y q (brazo largo) de cada cromosoma y se calculó el valor medio para cada par homólogo de cromosomas. Simultáneamente, se realizaron los siguientes cálculos: longitud relativa de cada par de cromosomas $=[$ longitud del cromosoma $\mathrm{p}+\mathrm{q} /$ longitud total del complemento cromosómico haploide (100)], proporción de brazos $(\mathrm{r}=\mathrm{q} / \mathrm{p})$, índice centromérico $[\mathrm{I} . \mathrm{C}=100(\mathrm{p} / \mathrm{p}+\mathrm{q})]$ y la diferencia entre brazos $[\mathrm{d}=\mathrm{r}-1(10) / \mathrm{r}+1]$. Dichos cálculos, se utilizaron para clasificar los cromosomas de acuerdo al criterio de Levan et al. (1964).

Debido a las dificultades para identificar los pares homólogos utilizando la clasificación de Levan et al. (1964), los cromosomas correspondientes al complemento cromosómico de ambas especies fueron divididos en cuatro grupos ("a", "b", "c" y "d") empleando las longitudes relativas de $\mathrm{p}+\mathrm{q}$. Los grupos fueron identificados del siguiente modo: grupo "a" birrámeos (10.0-7.80) único con brazos cortos $\mathrm{p}$ (pares del 1 al 3), grupo "b" (7.70-6.10) unirrámeos mayores (pares del 4 al 6), grupo "c" (6.09-4.10) monorrámeos medios (pares del 7 al 14) y grupo "d" (4.09-0.10) correspondiente a los monorrámeos menores (pares del 15 al 18) (Fig. 2, Cuadro 1).

Los datos ajustados a las longitudes relativas de cada par de cromosomas fueron analizados por las pruebas de normalidad y homocedasticidad $(P<0.05)$. A continuación, fueron comparados los pares cromosómicos de cada especie y entre especies ( $P$. columellaris vs $P$. pansa) por un análisis de varianza (ANVA) de dos vías y los valores significativos entre pares cromosómicos fueron identificados a $P<0.05$. Las diferencias en longitud entre pares cromosómicos, se observaron por la comparación múltiple de Tukey $(P<0.05)$.

El número fundamental (N.F) por especie fue establecido en función del número de cromosomas birrameos y el ideograma para cada 


\section{CUADRO 1}

Longitud promedio relativa por pares de cromosomas y estadísticas representativas del cariotipo de dos especies de caracol de tinte.

TABLE 1

Mean relative length, by chromosome pairs, and descriptive statistics from the karyotype of two purple snail species

$\begin{array}{ccc}\text { Par } & \text { Longitud } & \text { Longitud } \\ \text { Cromo- } & \text { relativa de } & \mathrm{r} \pm \mathrm{D} . \mathrm{E} \\ \text { sómico } & \mathrm{p} \pm \mathrm{D} . \mathrm{E}(\mu \mathrm{m}) & (\mu \mathrm{m})\end{array}$

$\begin{array}{lll}3.9 \pm 0.7(1.3) & 5.0 \pm 0.9(1.7) & 8.93 \pm 1.62^{\mathrm{a}^{*}} \\ 3.5 \pm 0.6(1.2) & 4.4 \pm 0.1(1.6) & 7.94 \pm 1.64^{\mathrm{a}^{*}} \\ 2.8 \pm 0.4(1.0) & 4.0 \pm 0.5(1.4) & 6.82 \pm 0.93^{\mathrm{a}^{*}} \\ & 7.5 \pm 0.9(2.6) & 7.52 \pm 0.88^{\mathrm{b}} \\ & 6.4 \pm 1.8(2.2) & 6.39 \pm 1.77^{\mathrm{b}} \\ & 6.3 \pm 0.7(2.2) & 6.33 \pm 0.75^{\mathrm{b}} \\ & 6.0 \pm 0.8(2.1) & 6.02 \pm 0.80^{\mathrm{c}} \\ & 5.8 \pm 0.6(2.0) & 5.87 \pm 0.63^{\mathrm{c}} \\ & 5.4 \pm 0.2(1.9) & 5.38 \pm 0.20^{\mathrm{c}} \\ & 5.3 \pm 0.2(1.8) & 5.30 \pm 0.23^{\mathrm{c}} \\ & 5.1 \pm 0.3(1.7) & 5.12 \pm 0.36^{\mathrm{c}} \\ & 4.9 \pm 0.3(1.7) & 4.93 \pm 0.33^{\mathrm{c}} \\ & 4.7 \pm 0.5(1.6) & 4.65 \pm 0.53^{\mathrm{c}} \\ & 4.5 \pm 0.5(1.5) & 4.52 \pm 0.57^{\mathrm{c}} \\ & 4.1 \pm 0.5(1.4) & 4.07 \pm 0.55^{\mathrm{d}} \\ & 3.9 \pm 0.6(1.3) & 3.86 \pm 0.61^{\mathrm{d}} \\ & 3.7 \pm 0.6(1.3) & 3.74 \pm 0.63^{\mathrm{d}^{*}} \\ & 3.2 \pm 0.5(1.1) & 3.28 \pm 0.56^{\mathrm{d}^{*}}\end{array}$
Longitud
relativa de $\mathrm{p}+\mathrm{q}$

$$
\begin{array}{ccc}
\begin{array}{c}
\text { Proporción } \\
\text { de brazos }
\end{array} & \begin{array}{c}
\text { Indice } \\
\text { centromérico }
\end{array} & \begin{array}{c}
\text { Diferencia } \\
\text { entre brazos }
\end{array} \\
\mathrm{r}=\mathrm{q} / \mathrm{p} & \mathrm{i}=100 \mathrm{p} / \mathrm{p}+\mathrm{q} & \mathrm{d}=\mathrm{r}-1(10) / \mathrm{r}+1
\end{array}
$$

Clasificación Grupos

P. pansa

$\begin{array}{lll}1.30 & 43.5 \pm 2.29 & 1.29 \\ 1.26 & 44.3 \pm 2.67 & 1.16 \\ 1.42 & 41.3 \pm 1.02 & 1.73 \\ & & \\ & \\ & \\ & \\ & \\ & \\ & \\ & \\ & \end{array}$

1.29

1.16
1.73

$\begin{array}{ll}\mathrm{m} & \mathrm{a} \\ \mathrm{m} & \mathrm{a} \\ \mathrm{m} & \mathrm{a} \\ \mathrm{t} & \mathrm{b} \\ \mathrm{t} & \mathrm{b} \\ \mathrm{t} & \mathrm{b} \\ \mathrm{t} & \mathrm{c} \\ \mathrm{t} & \mathrm{c} \\ \mathrm{t} & \mathrm{c} \\ \mathrm{t} & \mathrm{c} \\ \mathrm{t} & \mathrm{c} \\ \mathrm{t} & \mathrm{c} \\ \mathrm{t} & \mathrm{c} \\ \mathrm{t} & \mathrm{c} \\ \mathrm{t} & \mathrm{d} \\ \mathrm{t} & \mathrm{d} \\ \mathrm{t} & \mathrm{d} \\ \mathrm{t} & \mathrm{d}\end{array}$

P. columellaris

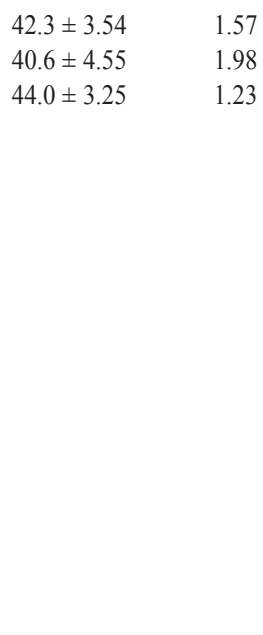

$\begin{array}{lll}1.37 & 42.3 \pm 3.54 & 1.57 \\ 1.49 & 40.6 \pm 4.55 & 1.98 \\ 1.28 & 44.0 \pm 3.25 & 1.23 \\ & & \\ & & \\ & \\ & \\ & \\ & \\ & \\ & \end{array}$

$\begin{array}{ll}\mathrm{m} & \mathrm{a} \\ \mathrm{m} & \mathrm{a} \\ \mathrm{m} & \mathrm{a} \\ \mathrm{t} & \mathrm{b} \\ \mathrm{t} & \mathrm{b} \\ \mathrm{t} & \mathrm{b} \\ \mathrm{t} & \mathrm{c} \\ \mathrm{t} & \mathrm{c} \\ \mathrm{t} & \mathrm{c} \\ \mathrm{t} & \mathrm{c} \\ \mathrm{t} & \mathrm{c} \\ \mathrm{t} & \mathrm{c} \\ \mathrm{t} & \mathrm{c} \\ \mathrm{t} & \mathrm{c} \\ \mathrm{t} & \mathrm{d} \\ \mathrm{t} & \mathrm{d} \\ \mathrm{t} & \mathrm{d} \\ \mathrm{t} & \mathrm{d}\end{array}$

D.E, desviación estándar; p, brazo corto; q, brazo largo; r, proporción de brazos; I.C, índice centromérico; d, diferencia entre brazos; $\mathrm{m}$, metacéntrico; $\mathrm{t}$, telocéntrico; $\mu \mathrm{m}$, micrometro.

a Literales iguales en pares cromosómicos análogos en cada especie indican diferencias no significativas $(P<0.05)$ y en caso contrario hay significancia.

* indica diferencias significativas $(P<0.05)$ entre especies. 
especie fue construido en orden decreciente y de acuerdo a la longitud relativa de cada par homologo de cromosomas (Denton 1973).

\section{RESULTADOS}

El análisis microscópico del tejido derivado de las gónadas de hembras y machos de ambas especies, no mostraron dispersiones cromosómicas. Sin embargo, en el caso del músculo y estómago, fueron los tejidos con mayor número de dispersiones cromosómicas en metafase. Los embriones, mostraron elevado número de células en metafase en relación con el tejido derivado de los adultos.

En $P$. pansa fueron contabilizados 186 campos mitóticos en metafase, 59 obtenidos de los ejemplares adultos y 127 provenientes de los embriones; de los cuales $42.3 \%$ y 21.2 $\%$ respectivamente mostraron número modal diploide de 36 cromosomas. También, se registraron dispersiones con número par e impar de cromosomas variando de los 30 y hasta los 54 cromosomas en los adultos y en los embriones fueron observados cromosomas desde $30 \mathrm{y}$ hasta los 49 elementos (Fig. 1 y 2).

En $P$. columellaris, se analizaron 118 campos mitóticos con $29.6 \%$ de las células contabilizadas con número modal diploide de 36 cromosomas (Fig. 1C). Simultáneamente, se registraron números cromosómicos pares e impares que variaron de los 17 y hasta los 45 cromosomas (Fig. 1C).

Los porcentajes de variación cromosómica observados en ambas especies fueron mas frecuentes en los números cromosómicos cercanos al número modal, siendo estos desde los 34 y hasta los 40 cromosomas (Fig. 1). Sin embargo, dicha variación no estuvo relacionada con los conteos observados en los tejidos empleados como fuente de dispersiones cromosómicas.

El complemento cromosómico diploide de $P$. pansa y $P$. columellaris fue clasificado de acuerdo con Levan et al. (1964); y se caracterizaron por tener los tres primeros pares de cromosomas del tipo metacéntricos (m) (Fig. 2 y 3, Cuadro 1). En ambas especies el resto de cromosomas (quince pares del 4 al 18) fueron clasificados como cromosomas telocéntricos (acrocéntricos) (Fig. 2 y 3, Cuadro 1).

El reducido tamaño y forma de punto de los cromosomas telocéntricos, dieron dificultades en la identificación de los pares homólogos por observación directa, no así cuando fueron utilizadas las longitudes relativas de cada cromosoma individual. Los pares cromosómicos dentro de cada grupo son en algunos casos difíciles de separar unos de otros. Sin embargo, las diferencias entre grupos de cromosomas son perceptibles si se emplea la propuesta de clasificacion por grupos recomendada en este documento.

Como se indica en las longitudes relativas de $p+q$ entre pares cromosómicos (Cuadro 1), el tamaño de los cromosomas representados por el grupo "c" y "d" fueron significativos (Tukey, $P=0.018,0.016$ ) en relación con los cromosomas del complemento cromosómico estándar de cada especie (grupos "a" y "b"). Por otro lado, las longitudes relativas a nivel intraespecifico (p.e. par 1 vs par 4) revelaron diferencias significativas (Tukey, $P=0.008$ ) en el complemento cromosómico de cada especie. La comparación de las longitudes relativas $(\mathrm{p}+\mathrm{q})$ entre especies, mostraron que los pares cromosómicos 1, 2, 3, 17 y 18 de $P$. pansa fueron significativos en relación con los de $P$. columellaris, mientras que los pares del 4 al 16 no lo fueron (Tukey, $P<0.05$ ) (Cuadro 1). No fueron identificados cromosomas adicionales con tamaño y apariencia similar a la de los cromosomas del grupo "a" y "b". El número fundamental (N.F) característico en ambas especies fue de 42 brazos cromosómicos. En los cariotipos de hembras y machos no fue posible observar diferencias heteromórficas que permitieran caracterizar cromosomas sexuales en las especies examinadas (Fig. 2). 

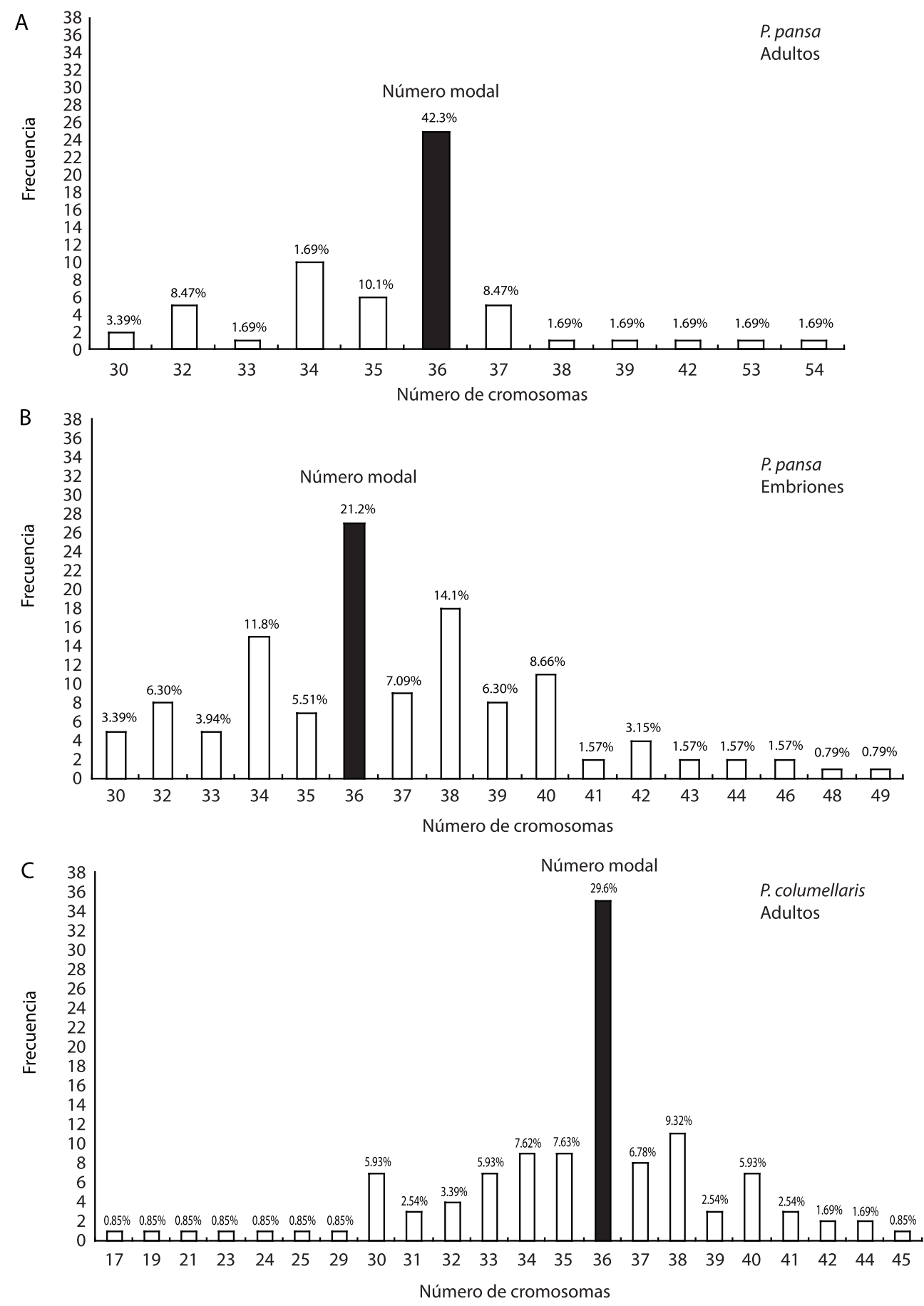

Fig. 1. Número modal diploide de $2 \mathrm{n}=36$ cromosomas, frecuencia de conteos en porcentajes en adultos (A) y embriones (B) de $P$. pansa y en adultos de P. columellaris (C).

Fig. 1. Diploid modal number of $2 \mathrm{n}=36$ chromosomes; count frequency for percentages in adults (A) and embryos (B) of $P$. pansa and in adults of $P$. columellaris $(\mathrm{C})$. 


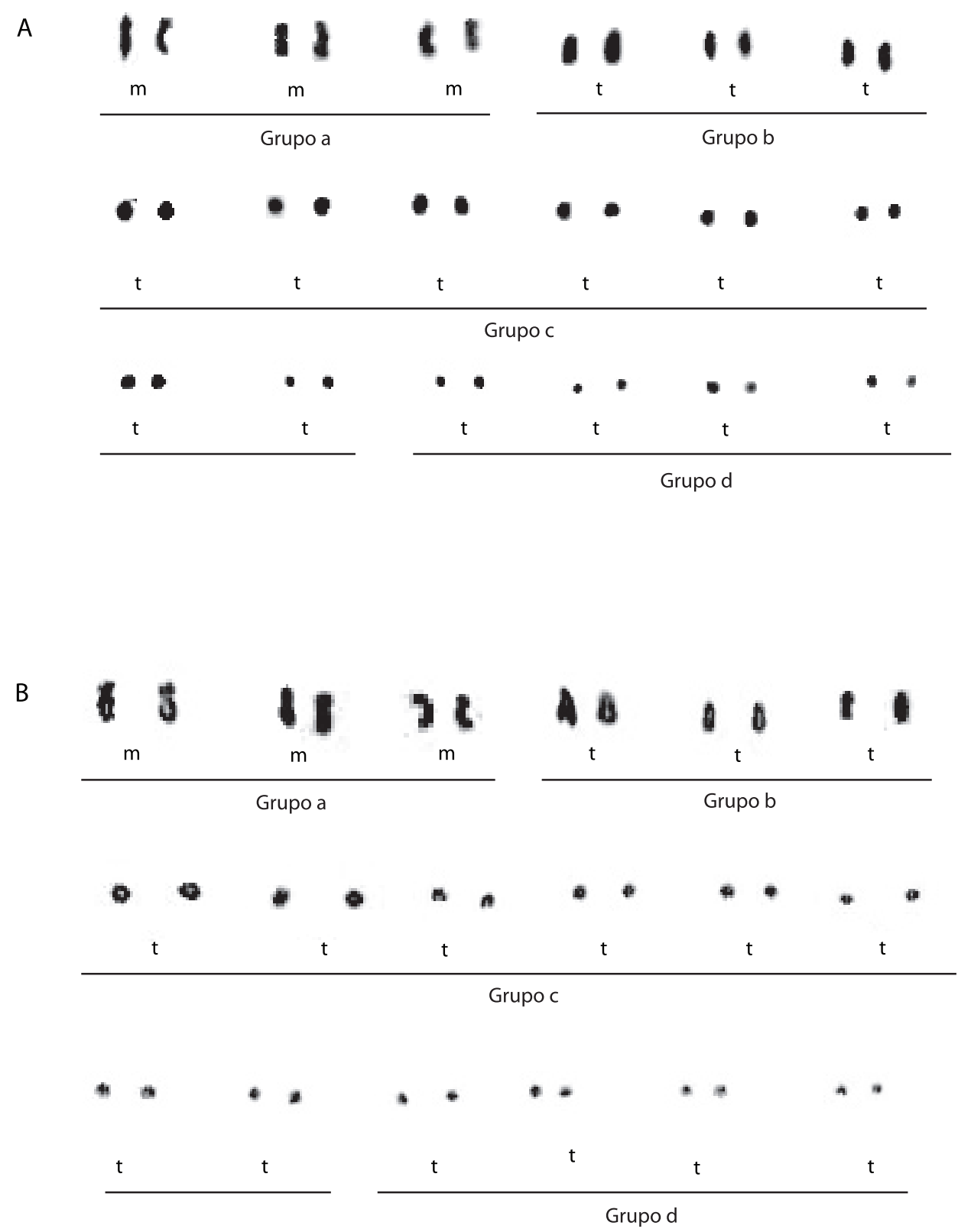

Fig. 2. Cariotipos de los caracoles de tinte $P$. pansa (A) y P. columellaris (B), representados por $2 \mathrm{n}=36$ cromosomas, clasificados en dos categorías ( $\mathrm{m}=$ metacéntrico y $\mathrm{t}=$ telocéntrico) y divididos en cuatro grupos ("a", "b", "c" y “d”).

Fig. 2. Karyotypes of purple snail P. pansa (A) and P. columellaris (B) characterized by $2 \mathrm{n}=36$ chromosomes, classified in two types ( $\mathrm{m}=$ metacentric and $\mathrm{t}=$ telocentric) and divided in four groups ("a", "b", "c" and "d"). 

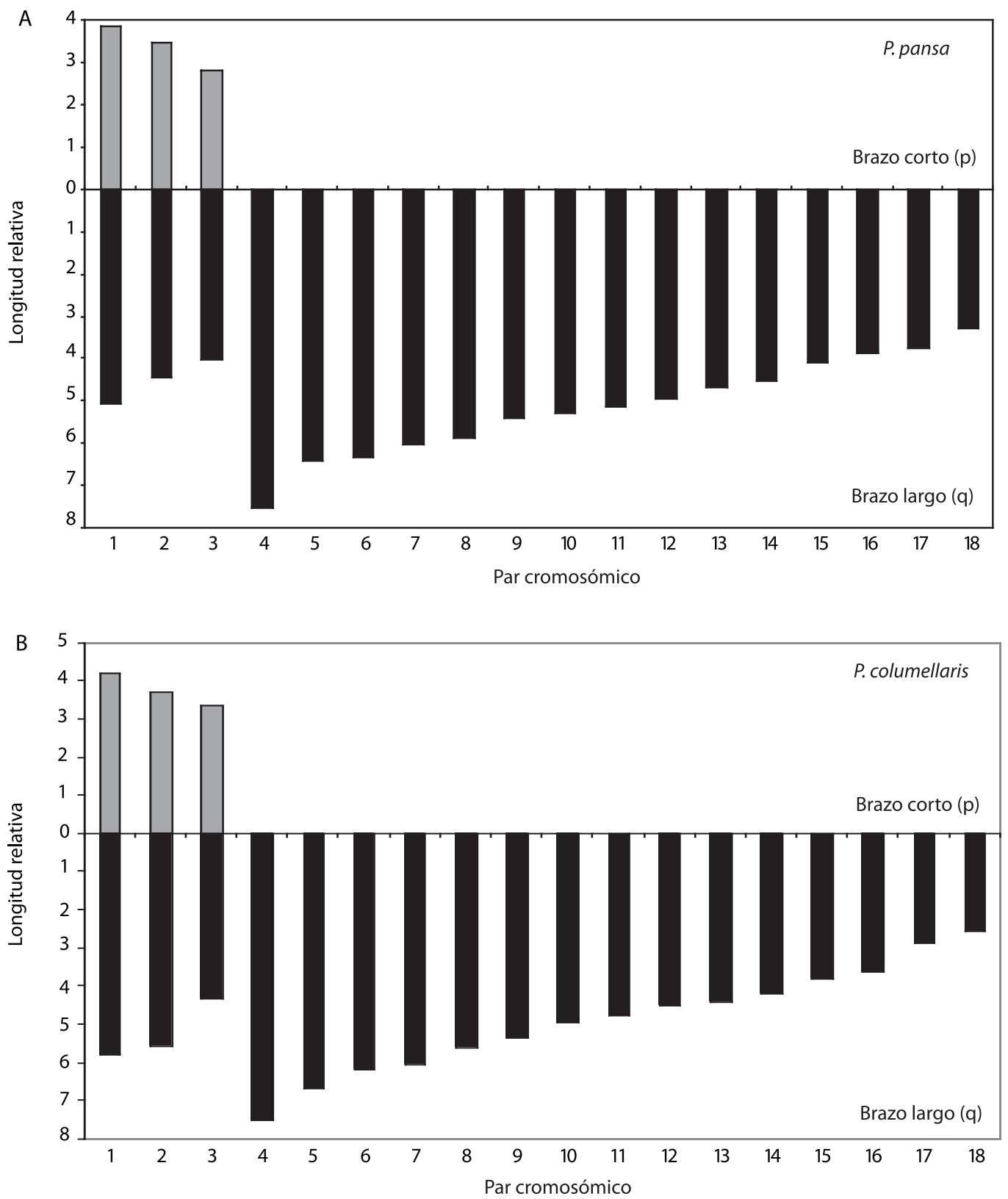

Fig. 3. Ideogramas representativos del complemento cromosómico promedio por pares, de los cariotipos de $P$. pansa (A) y P. columellaris (B).

Fig. 3. Representative ideograms from the averaged chromosome complement by pairs of the karyotype of $P$. pansa (A) and P. columellaris (B). 


\section{DISCUSIÓN}

Los números cromosómicos en los adultos y embriones de $P$. pansa mostraron 36 elementos como número modal diploide, siendo este similar a los conteos de $P$. columellaris. La variación cromosómica identificada a nivel intra-especifico y por individuo, coinciden con los reportados en N. lapillus (Page 1988, Dixon et al. 1994, Pascoe y Dixon 1994, Pascoe et al. 1996). En P. pansa y P. columellaris, se observaron conteos cromosómicos inferiores y superiores a los 36 elementos que integran el cariotipo (Fig. 1).

La variación cromósomica intra-individual (variación del número de cromosomas en los diferentes tejidos de cada espécimen) e inter-individual (variación del número de cromosomas entre individuos de la misma especie) fue notable en ambas especies y no estuvo relacionada con el tipo de tejido utilizado en los especímenes adulto (Fig. 1A y C). Por lo que se descarta un fenómeno interno de poliploidización en cada tejido. Las diferencias identificadas en los números cromosómicos de $P$. pansa y $P$. columellaris podrían suponer conteos cromosómicos particulares en cada individuo. Sin embargo, es muy probable que cada espécimen mantenga estabilidad cromósomica tanto en el número de elementos como en el tipo (Fig. 1 y 2).

Los números cromosómicos aquí reportados, posiblemente son el resultado de uno o de la combinación de varios fenómenos. El polimorfismo cromosómico que en el orden Neogastropoda al que pertenecen estas especies solo ha sido reportado en $N$. lapillos, como resultado de varios eventos en los que participan fusiones o fisiones cromosómicas simples que afectan ha cinco pares de cromosomas metacéntricos (Staiger 1954, Pascoe y Dixon 1994, Pascoe et al. 1996). Algunos estudios arrojan evidencias que muestran variación inter-individual e intra-individual en el número cromosómico de poblaciones en las que existe polimorfismo (Dixon et al. 1994) y en las que ocurren rearreglos cromosómicos estructurales con frecuencia (Page 1988).
La morfología y las longitudes relativas de los cromosomas de $P$. pansa y $P$. columellaris, son estrechamente semejantes de acuerdo con la formula cromósomica de los cariotipos tipo: $6 \mathrm{~m}+30 \mathrm{t}$, el N.F=42 y la división por grupos ("a", "b", "c" y "d") sugerida para el complemento cromosómico de cada especie. Sin embargo, los resultados del análisis estadístico indica que los tres primeros pares de cromosomas (grupo "a") y dos últimos (grupo "d") en ambas especies manifiestan diferencias significativas (Tukey, $P<0.05$ ) en las longitudes relativas de $\mathrm{p}+\mathrm{q}$ (Cuadro 1 ).

Los efectos derivados de la colchicina y las consecuencias de la acción de los fijadores sobre los cromosomas (principalmente del ácido acético) pueden reducir el tamaño de los cromosomas (Denton 1973, Kligerman y Bloom 1977, Henegariu et al. 2001). También, el tamaño obedece al grado de avance de la metafase y finalmente el juicio utilizado para determinar la posición presuntiva del centrómero y medir la talla de los brazos en cada cromosoma (Arias-Rodriguez et al. 2006).

En este estudio, los organismos fueron tratados con una concentración estándar de colchicina, atendiendo básicamente al régimen biomasa o gramos del espécimen y el tiempo exacto de exposición al agente, por lo que se podría esperar un efecto homogéneo sobre la cromatina y el tamaño de la mayoría de los campos cromosómicos. Aunque, el tiempo de exposición al agente mitostatico fue particularmente prolongado si se compara con el empleado en otros grupos taxonómicos (de 4 a 6 h).

Experimentos previos (datos no mostrados) en los caracoles de tinte permitieron establecer que una exposición de corto y mediano plazo (de 3-8 h) no afecta significativamente el ritmo de división celular por lo que no fue posible observar campos cromosómicos. Sin embargo, si el tiempo de exposición al alcaloide se prologa por $10 \mathrm{~h}$, abundante número de dispersiones cromosómicas en metafase son observadas en varios tejidos pero no en las gónadas, siguiendo el protocolo que se recomienda en el presente estudio. Los caracoles son organismos con un ritmo de vida considerada lenta, por lo que es 
posible que los ciclos de división celular estén regulados por tal comportamiento y para detener la división celular es necesario incrementar los tiempos de exposición al agente mitostatico como el empleado en este estudio. Aunque el efecto puede variar dependiendo de la especie, por ejemplo en el caracol de agua dulce Pomacea catemacensis dos horas de exposición a la colchicina fueron los necesarios para obtener dispersiones de cromosomas de buena calidad y en metafase (Diupotex-Chong et al. 2004).

Las diferencias identificadas únicamente en los cromosomas metacéntricos (grupo "a") y los últimos dos pares de cromosomas monorrámeos del grupo "d"; sugieren que las desproporciones (variaciones en las longitudes relativas) sean el resultado de un probable intercambio azaroso de bloques grandes de cromatina o microcromosomas entre ambos tipos de cromosomas. Si existen rearreglos estructurales entre los cromosomas metacéntricos y los telocéntricos del complemento cromosómico de $P$. pansa y $P$. columellaris, los cambios se deberían reflejar como variaciones tanto en el número de cromosomas, como en las longitudes de los mismos, tal y a como se indica en los resultados de este documento y los de $N$. lapillus (Page 1988, Dixon et al. 1994, Pascoe y Dixon 1994, Pascoe et al. 1996).

En acuerdo con Jones y Rees (1982) y Beukeboom (1994), los cromosomas "B" pueden ser distinguidos con facilidad de los cromosomas normales (tipo o estándar) por las siguientes condiciones: reducido tamaño en comparación con el resto de cromosomas, variación numérica intra-individual e inter-individual, heterocromáticos (bandas-C positivas), alta frecuencia en células meióticas, sin emparejamiento ( $\sin$ recombinación) en meiosis I.

Los microcromosomas o cromosomas " $\mathrm{B}$ " en el presente documento cumplen con dos de las condiciones señaladas, a excepción de las bandas-C positivas y del análisis de dispersiones cromosómicas en meiosis que no fue posible estudiar en este trabajo. El origen de los microcromosomas " $\mathrm{B}$ " tiene varias explicaciones de acuerdo con la literatura, una de ellas es el origen intraespecifico a partir del complemento cromosómico "A" estándar o el origen ínterespecifico como resultado de la hibridación entre especies cercanas (Camacho et al. 2000).

El daño al genoma por origen antropogénico ha sido mencionado con frecuencia en la literatura especializada (Amorin et al. 2000, Baršien 2003, Nakano et al. 2003). Es bien conocido que el mercurio y sus derivados interfieren con el huso durante la división celular (Miura y Imura 1987) y pueden contribuir con el aislamiento de fragmentos de ADN durante la división de la célula, como ha sido reportado para los habitantes del Amazonas expuestos a contaminación por metilo de mercurio (Amorim et al. 2000). En cuatro especies de caracoles del género Viviparus se observaron daños a nivel citogenético con prevalecía de células poliploides en algunos tejidos de adultos y embriones como resultado de la exposición a genotóxicos disueltos en varios lagos de suiza (Baršien 2003).

La explotación de minas de oro, plata, manganeso y cobre en los afluentes adyacentes a la zona de muestreo, junto con el empleo de mercurio y otros compuestos químicos adecuados para la extracción y purificación de los metales, podrían ser una fuente potencial de daño al genoma de $P$. pansa y $P$. columellaris que debería ser evaluada en el futuro. En lo que concierne a una posible hibridación entre las especies del género Plicopurpura no debe descartarse dado que hay coexistencia entre los hábitats de $P$. pansa y $P$. columellaris, pero no existe evidencia que pueda avalar dicha hipótesis a excepción de los resultados señalados en este estudio.

En conclusión, la eventualidad de que las diferencias en las longitudes relativas $(p+q)$, se tratase de indicadores que nos hagan suponer divergencia especifica entre $P$. pansa y $P$. columellaris tienen soporte en el trabajo de Castillo-Rodriguez (1992a), donde señala diferencias en los caracteres conquiliológicos, radulares y en la estructura del tracto digestivo entre $P$. pansa, $P$. columellaris y $P$. patula.

No obstante y en acuerdo con el mismo autor, las especies de que trata este estudio 
conservan un patrón común, anatómica y con los datos que aporta este trabajo citogenéticamente (Fig. 2 y 3 ) que evidentemente las podría ubicar dentro de una unidad taxonómica propia.

A pesar de ello, es necesario que en el futuro se realicen estudios comparativos más finos como son el uso de la tinción diferencial de cromosomas (bandas C, G y R) que en algunas especies de invertebrados marinos ha resultado ser refractaria (Pascoe y Dixon 1994, Pascoe et al. 1996), no así el uso de la tinción con nitrato de plata para la localización de los organizadores nucleolares (NORs), la hibridación In Situ fluorescente (FISH) y las sondas moleculares de $\mathrm{ADNr}$ con las que se ha logrado elucidar las relaciones entre el número cromosómico y las condiciones ambientales que promueven el polimorfismo cromosómico (Pascoe y Dixon 1994, Pascoe et al. 1996, Kirby 2000).

La aplicación de dichas herramientas en conjunto con algunas técnicas de biología molecular han sido de mucha utilidad en otras especies (Rawlings et al. 2001, Boore et al. 2004, Grande et al. 2004) y podrían ser muy valiosas para el esclarecimiento y separación de las poblaciones de $P$. pansa, $P$. columellaris y $P$. patula que cohabitan en la región sur de las costas del Pacífico, en el continente Americano.

\section{AGRADECIMIENTOS}

Este trabajo fue realizado con financiamiento parcial del Centro de Investigación en Alimentación y Desarrollo, A.C. Unidad Mazatlán (Proyecto Fiscal: 2001-6250-1), del Fondo Mexicano para la Conservación de la Naturaleza A.C. (AMACUP) (Proyecto: A-2-98-14) y de la Universidad Juárez Autónoma de Tabasco. Los autores agradecen las facilidades otorgadas por Emma Josefina Fajer Avila, responsable del laboratorio de parasitología CIAD-Mazatlán para el empleo del equipo de microfotografía digital.

\section{RESUMEN}

El cariotipo de Plicopurpura pansa y P. columellaris fue determinado a partir de 17 y 13 especímenes adultos respectivamente. Adicionalmente, se utilizaron ocho cápsulas de P. pansa. Contamos 186 campos mitóticos en P. pansa: 59 en los adultos y 127 en los embriones; y 118 campos en P. columellaris. En ambas especies se observaron números cromosómicos desde 30 hasta 42 . Las variaciones en número cromosómico fueron identificadas en cada individuo, no habiendo ninguna relación con los tejidos (branquias, músculo y estómago) empleados. El número modal diploide típico fue de $2 \mathrm{n}=36$ cromosomas en ambas especies. En los especímenes adultos seleccionamos cinco de las mejores dispersiones cromosómicas para armar el cariotipo. Calculamos los estadísticos citogenéticos clásicos, longitudes relativas, proporción de brazos, índice centromérico y la diferencia entre brazos. Identificamos en ambas especies tres pares de cromosomas metacéntricos y quince pares de cromosomas telocéntricos. Esta clasificación no fue suficientemente robusta, por lo que dividimos el complemento cromosómico de cada especie en cuatro grupos ("a", "b", "c" y "d") utilizando como criterio las longitudes relativas $(p+q)$. Hubo diferencias significativas entre grupos cromosómicos por especie y entre especies, los tres primeros pares de cromosomas (grupo "a" birrámeos) y los dos últimos pares (grupo "d" monorrámeos menores) (análisis de varianza de dos vías, $\mathrm{p}<0.05$ ). Las desviaciones en número cromosómico y en las longitudes relativas, posiblemente se deban a reorganizaciones cromosómicos asociadas con el polimorfismo cromosómico y presencia de microcromosomas tipo "B". El número fundamental en ambas especies se caracterizó por 42 brazos cromosómicos. No identificamos cromosomas sexuales.

Palabras clave: Plicopurpura, pansa, columellaris, cromosomas, polimorfismo.

\section{REFERENCIAS}

Abbott, R.T. 1974. American seashells. Van Nostrand Reinhold. Nueva York, Nueva York, EEUU.

Acevedo, G.J. \& C.E. Hernández. 1987. Aspectos poblacionales y etnobiológicos del caracol Purpura pansa (Gould 1853) en la costa de Oaxaca. Tesis de Licenciatura, Universidad Nacional Autónoma de México, México, D.F., México.

Ahmed, M. 1974. Chromosome variation in three species of the marine gastropod Nucella. Cytologia 39: 597-607.

Álvarez, A. 1989. Relaciones ecológicas y algunos aspectos poblacionales del caracol Purpura pansa Gould, 1853 en la costa del estado de Michoacán, México. Tesis de Licenciatura, Universidad Michoacana, San Nicolás de Hidalgo, Michoacán, México.

Amorim, M.I., D. Mergler, M.O. Bahia, H. Dubeau, D. Miranda, J. Lebel, R.R. Burbano \& M. Lucotte. 2000. 
Cytogenetic damage related to low levels of methyl mercury contamination in the Brazilian Amazon. An. Acad. Bras. Cienc. 72: 487-507.

Arias-Rodriguez, L., S. Páramo-Delgadillo \& A.L. DuránGonzález. 2006. Caracterización citogenética del pez tropical de agua dulce Parachromis managuensis (Pisces: Cichlidae). Rev. Biol. Trop. 54: 35-42.

Baršien, J. 2003. Cytogenetic damage in viviparid snails inhabiting different lakes in Switzerland. Ekologija 3: 23-28.

Beukeboom, L.W. 1994. Bewildering Bs: an impression of the 1st B-chromosome conference. Heredity 73 : $328-336$

Boore, J.L., M. Medina \& L.A. Rosenberg. 2004. Complete sequences of the highly rearranged molluscan mitochondrial genomes of the Scaphopod Graptacme eborea and the bivalve Mytilus edulis. Mol. Biol. Evol. 21: 1492-1503.

Camacho, J.P.M., T.F. Sharbel \& L.W. Beukeboom. 2000 B-chromosome evolution. Phil. Trans. R. Soc. Lond B. $355: 163-178$.

Castillo-Rodriguez, Z.G. 1992a. Combinatio nova de Plicopurpura pansa (Gould, 1853) (Prosobranchia: Muricoidea). An. Inst. Cienc. Del Mar y Limnol. U.N.A.M. 19: 103-111.

Castillo-Rodriguez, Z.G. \& F. Amezcua-Linares. 1992b. Biología y aprovechamiento del caracol morado Plicopurpura pansa (Gould, 1853) (Gastropoda: Neogastropoda) en la costa de Oaxaca, México. An. Inst. Cienc. Del Mar y Limnol. U.N.A.M. 9: 223-234.

Cernohorsky, W.O. 1969. The Muricidae of Fiji, Part II -Subfamily Thaidinae. Veliger 11: 293-315.

Clench, W.J. 1947. The genera Purpura and Thais in the western Atlantic. Johnsonia 2: 61-75.

Denton, T.E. 1973. Fish chromosome methodology. Charles C. Tomas, Chicago, Illinois, EEUU.

Dixon, D.R., P.L. Pascoe, P.E. Gibbs \& J.J. Pasantes. 1994. The nature of Robertsonian chromosomal polymorphism in Nucella lapillus: a re-examination, p 389-399. In A.R. Beaumont (ed.). Genetics and evolution of aquatic organisms. Chapman and Hall, Londres, Inglaterra.

Diupotex-Chong, M.E., N.J. Cazzaniga, A. HernandezSantoyo \& J.M. Betancourt-Rule. 2004. Karyotype description of Pomacea patula catemacensis (Caenogastropoda, Ampullariidae), with an assessment of the taxonomic status of Pomacea patula. Biocell. 28: 279-285.

Fagundes, V., J.P.M. Camacho \& Y. Yonenaga-Yassuda. 2004. Are the dot-like chromosomes in Trinomys iheringi (Rodentia, Echimyidae) B chromosomes? Cytogenet. Genome Res. 106: 159-164.

Granado, N., E. Rebollo, F.J. Sánchez \& P. Arana. 2004. B chromosomes: the troubles of integration. Cytogenet. Genome Res. 106: 402-410.

Grande, C., J. Templado, J.L. Cervera \& R. Zardoya. 2004. Molecular phylogeny of Euthyneura (Mollusca: Gastropoda). Mol. Biol. Evol. 21: 303-313.

Henegariu, O., N.A. Heerema, L.L. Wright, P. Bray-Ward, D.C. Ward \& G.H. Vance. 2001. Improvements in cytogenetic slide preparation: controlled chromosome spreading, chemical and gradual denaturing. Cytometry 43: 101-109.

Jones, R.N. \& H. Rees. 1982. B Chromosomes. Academic, Londres, Inglaterra.

Keen, M. 1971. Sea shells of tropical west America. Stanford University, Stanford, California, EEUU.

Kirby, R.R. 2000. An ancient transpecific polymorphism shows extreme divergence in a Multitrait cline in an intertidal snail (Nucella lapillus) (L.). Mol. Biol. Evol. 17: 1 816-1 825.

Kligerman, A.D. \& S.E. Bloom. 1977. Rapid chromosome preparation from solid tissues of fishes. J. Fish. Res. Board Can. 34: 266-269.

Kool, S.P. 1987. Significance of radular characters for reconstruction of thaidid phylogeny (Neogastropoda: Muricacea). Nautilus 101: 117-131.

Kool, S.P. 1988. Aspects of the anatomy of Plicopurpura patula (Prosobranchia: Muricoidea: Thaidinae), new combination, with emphasis on the reproductive system. Malacología 29: 373-382.

Levan, A., K. Fredga \& A.A. Sandberg. 1964. Nomenclature for centromeric position of chromosomes. Hereditas 52: $201-220$.

Michel-Morfín, J.E., E.A. Chávez \& L. González. 2002. Estructura de la población, esfuerzo y rendimiento de tinte del caracol Plicopurpura pansa (Gould, 1853) en el Pacífico Mexicano. Ciencias Marinas 28: $357-368$. 
Michel-Morfín, J.E., E.A. Chávez \& V. Landa. 2000. Population parameters and dye yield of the purple snail Plicopurpura pansa in Oaxaca; (Gould, 1853) of west central México. J. Shellfish Res. 19: 919-925.

Miura, K. \& N. Imura. 1987. Mechanism of methyl mercury toxicity. CRC Crit. Rev. Toxicol. 18: 161-187.

Moreira-Filho, O., Jr., P.M. Galetti \& L.A.C. Bertollo. 2004. B chromosomes in the fish Astyanax scabripinnis (Characidae, Tetragonopterinae): An overview in natural populations. Cytogenet Genome Res. 106: 230-234.

Naegel, L.C.A. 2004. Laboratory spawning of the purple snail Plicopurpura pansa (Gastropoda: Muricidae). Rev. Biol. Trop. 52: 57-65.

Palestis, B.G, R. Trivers, A. Burt \& R.N. Jones. 2004. The distribution of $\mathrm{B}$ chromosomes across species. Cytogenet Genome Res.106:151-158.

Palestis, B.G, A. Burt, R.N. Jones \& R. Trivers. 2004. B chromosomes are more frequent in mammals with acrocentric karyotypes: support for the theory of centromeric drive. Proc. Biol. Sci. 271: S22-S24.

Page, C. 1988. The chromosome complement of Nucella lapillus (L.), Mollusca: Gastropoda: Prosobranchia. Caryologia 41: 79-91.

Pascoe, P.L. \& D.R. Dixon. 1994. Structural polymorphism in the dogwhelk Nucella lapillus (Mollusca: Neogastropoda). Mar. Biol. 118: 247-253.

Pascoe, P.L., S.J. Patton, R. Critcher \& D.R. Dixon. 1996 Robertsonian polymorphism in the marine gastropod, Nucella lapillus: advances in karyology using rDNA and NORs. Chromosoma 104: 455-460.

Ramírez-Rodríguez, M. \& L.C.A. Naegel. 2003. Crecimiento del caracol de tinte Plicopurpura pansa en Baja California Sur, México. Ciencias Marinas 29: $283-290$.

Rawlings, T.A., T.M. Collins \& R. Bieler. 2001. A major mitochondrial gene rearrangement among closely related species. Mol. Biol. Evol. 18: 1 604-1 609.

Rebollo, E. \& P. Arana. 2001. Active role of lagging chromosomes in spindle collapse as revealed by live phase contrast and tubulin immunostaining in grasshopper spermatocytes. Chromosoma 110: 292-304.

Rios-Jara, E., H.G. León-Alvarez, L. Lizárraga-Chávez \& J.E. Michel-Morfín. 1994. Producción y tiempo de recuperación del tinte de Plicopurpura patula pansa (Neogastropoda: Muricidae) en Jalisco, México. Rev. Biol. Trop. 42: 537-545.

Staiger, H. 1954. Der chromosomen dimorhismus beim Prosobranchier Purpura lapillus in Bezienhung zur ökologie der Art. Chromosoma 6: 419-478.

Thiriot-Quiévreux, C. 2003. Advances in chromosomal studies of gastropod Mollusks. J. Moll. Stud. 69: $187-201$.

Thorgaard, G. \& J. Disney. 1990. Chromosome preparation and analysis, p 172-187. In C. Schreck \& P. Moyle (eds.). Methods for fish biology. American Fisheries Society, Maryland, EE.UU.

Turok, M., A. Sigler-M, E. Hernández-C, J. Acevedo-R, R. Lara-C \& V. Turcott. 1988. El caracol Púrpura una tradición milenaria en Oaxaca. Dirección General de Culturas populares, SEP, México.

Vitturi, R., M.B. Rasotto \& N. Farinella-Ferruzza. 1982. The chromosomes of 16 molluscan species. Bollettino di Zoologia 49: 61-71.

Wellington, G.M. \& A. Kuris. 1983. Growth and shell variation in the tropical eastern Pacific intertidal gastropod genus Purpura: ecological and evolutionary implications. Biol. Bull. 164: 518-535.

White, M.J. 1954. Animal cytology. and Evolution. Cambridge Univ., Londres, Inglaterra.

Wouters, J. 1992. A new method for the analysis of blue and purple dyes in Textiles. Dyes Hist. Archaeol. 10: $17-21$.

\section{REFERENCIAS DE INTERNET}

Turok, M. 1999. El caracol Purpura pansa: Un pequeño gran recurso. Programa Cultural Tierra Adentro (consultado 2 noviembre 2005, http://www.cnca.gob. $\mathrm{mx} / /$ cnca/buena/descentra/tierra/ mturok.html). 